\title{
Analysis of copy number variations of WNT4 gene in a Chinese population with Müllerian anomalies
}

Ying Zhu ${ }^{1,2,3 \dagger}$, Ruyi Wang ${ }^{4,5 \dagger}$, Yun Cheng ${ }^{1,2,3}$, Yang Han ${ }^{1,2,3}$, Tengyan $\mathrm{Li}^{5}$, Yunxia Cao ${ }^{1,2,3^{*}}$ and Binbin Wang ${ }^{4,5^{*}}$ (0)

\begin{abstract}
Background: To investigate the genetic contribution of copy number variations (CNVs) in Wingless-type MMTV integration site family, member 4 (WNT4), in a Chinese population with Müllerian anomalies (MA), copy number analysis of WNT4 by Multiplex ligation-dependent probe amplification (MLPA) was performed on 248 female patients. Some studies have shown that heterozygous missense mutation of WNT4 can lead to MA. However, few studies on the relationship between WNT4 CNVs and MA have been performed.
\end{abstract}

Results: Among the 248 Chinese women affected by MA in this study, heterozygous deletion of WNT4 was detected in a single patient.

Conclusions: MLPA identified one heterozygous deletion in WNT4 in a single female patient among 248 Chinese women affected by MA. This study firstly reports CNVs of WNT4 in a large sample of MA patients from the Chinese population, which suggests that CNVs of WNT4 cannot be excluded in the occurrence of MA. This provides a genetic basis for precise treatment in the future.

Keywords: Copy number variations, WNT4, Müllerian anomalies, Chinese population

\section{Background}

Müllerian anomalies (MA) are among the most common diseases in gynecology and obstetrics. They include various malformations of fallopian tubes, vagina, cervix, and corpus uteri [1]. The estimated incidence of MA is $5.5 \%$ in the general population, but $24.5 \%$ in women with infertility and miscarriage $[2,3]$. This disease seriously affects women's reproductive function and causes a huge social burden.

According to guidance of the European Society of Human Reproduction and Embryology/European Society

\footnotetext{
*Correspondence: caoyunxia6@126.com; wbbahu@163.com

${ }^{\dagger}$ Ying Zhu and Ruyi Wang considered as joint first authors

1 Reproductive Medicine Center, Department of Obstetrics

and Gynecology, The First Affiliated Hospital of Anhui Medical University,

218 Jixi Road, Shushan, Hefei 230022, China

${ }^{4}$ Graduate School of Peking Union Medical College, Beijing, China

Full list of author information is available at the end of the article
}

for Gynaecological Endoscopy (ESHRE/ESGE) in 2013 [4], Müllerian duct abnormalities can be classified into 36 different combinations as the main class for the uterine body $(\mathrm{U})$ and subclasses for the cervix $(\mathrm{C})$ and vagina (V). Compared to the classification method put forward by American Fertility Society, ESHRE/ESGE classified uterine morphology into 7 classes: U0, normal; U1, dysmorphic; U2, septate; U3,bicorporeal; U4, hemiuterus; U5, aplastic; U6, unclassified cases.

Different types of Müllerian duct anomalies have different effects on reproductive function. People with mild symptoms can have normal fertility, but most patients with uterine malformations exhibit serious effects such as primary amenorrhea, recurrent abortion, and infertility. Septate uterus is one of the milder symptoms in patients with uterine malformation, and the rate of live births from such patients is about $62.5 \%$. After operation, this rate can be significantly increased [5].

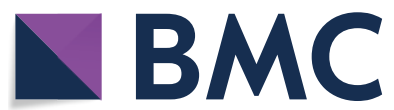

(c) The Author(s) 2021. This article is licensed under a Creative Commons Attribution 4.0 International License, which permits use, sharing, adaptation, distribution and reproduction in any medium or format, as long as you give appropriate credit to the original author(s) and the source, provide a link to the Creative Commons licence, and indicate if changes were made. The images or other third party material in this article are included in the article's Creative Commons licence, unless indicated otherwise in a credit line to the material. If material is not included in the article's Creative Commons licence and your intended use is not permitted by statutory regulation or exceeds the permitted use, you will need to obtain permission directly from the copyright holder. To view a copy of this licence, visit http://creativecommons.org/licenses/by/4.0/. The Creative Commons Public Domain Dedication waiver (http://creativecommons.org/publicdomain/zero/1.0/) applies to the data made available in this article, unless otherwise stated in a credit line to the data. 
To date, many genes related to MA have been found, including many genes of the Wingless-type MMTV integration site family (WNT4, WNT5A, WANT7, WNT9B) [6-10]. WNT4 (OMIM: 603490), a transcriptional regulator, plays an important role in sex determination, nephrogenesis, and female reproductive tract development in the early embryo [6, 11-13]. WNT4 ${ }^{-1-}$ female mice were reported to show phenotypes of sex reversal, Müllerian duct aplasia, hyperandrogenism, and follicle depletion [6, 14].

Many studies have also shown that the heterozygous missense mutation of WNT4 can lead to MA and hyperandrogenism in humans [15-18]. However, few studies have been performed on the relationship between WNT4 copy number variations (CNVs) and MA. Therefore, here we detected the copy number of WNT4 in 248 female patients with MA.

\section{Materials and methods Participants}

248 unrelated MA patients were recruited from the Reproductive Medicine Center of the First Affiliated Hospital of Anhui Medical University. All patients were Chinese, ranging in age from 22 to 48 years $(29.32 \pm 4.87)$. All patients showed a normal karyotype of $46, \mathrm{XX}$ and had been diagnosed with abnormal Müllerian ducts using physical examination, ultrasonographic investigations, hysteroscopy and laparoscopy. The study protocol was approved by the Ethics Committee of the National Research Institute for Family Planning, and written informed consent was obtained from all participants.

\section{DNA extraction}

Genomic DNA was extracted from the peripheral blood of 248 patients using the QIAamp DNA Blood Mini Kit (Qiagen, Hilden, Germany).

\section{MLPA assay}

Three probes designed in-house targeted the region of the WNT4 gene. The first probe covered exon 1 and exon 2, second probe covered exon 3 and exon 4, third probe covered exon 5. Fluorescence data on the PCR products analyzed by capillary electrophoresis were collected using an Applied Biosystems 3730xl DNA Analyzer, and the peak area data were output. Relative peak area of WNT4 was compared with that of the reference gene. Dosage quotient (DQ) was used as the signal result of CNVs. The classification was as follows: normal (0.8-1.2), homozygous deletion (0), heterozygous deletion (0.4-0.65), and heterozygous duplication (1.3-1.65).

\section{Analysis of CNV pathogenicity}

The pathogenicity of CNVs was evaluated in accordance with the $\mathrm{CNV}$ interpretation scoring metric from the American College of Medical Genetics and Genomics (ACMG) [19]. Online databases including the Database of Genomic Variants (DGV, http://dgv.tcag.ca/dgv/app/ home/) and the Genome Aggregation Database (GnomAD, http://gnomad.broadinstitute.org/) were used to retrieve the frequency of CNVs. Public databases, including PubMed (https://pubmed.ncbi.nlm.nih.gov/) and Online Mendelian Inheritance in Man (OMIM, https:// www.omim.org/), were used to evaluate the significance of CNVs of WNT4.

\section{Results \\ MLPA results of MA patients}

One deletion was identified in WNT4 in a single female patient among the 248 Chinese women affected by MA in this study (Fig. 1). The DQ values of three probe recognition sequences were all between 0.4 and 0.65 , reflecting a heterozygous deletion of WNT4 (Table 1).

\section{Clinical characteristics of the patient with CNVs}

The patient with a single copy of WNT4 was 28 years old, had regular menstruation, and exhibited normal levels of estradiol (E2) and testosterone (T). She suffered from secondary infertility, having been diagnosed with partial septate uterus but no renal abnormalities. She had undergone transcervical incision of septa (TCIS) in 2016 (see Table 1 for details).

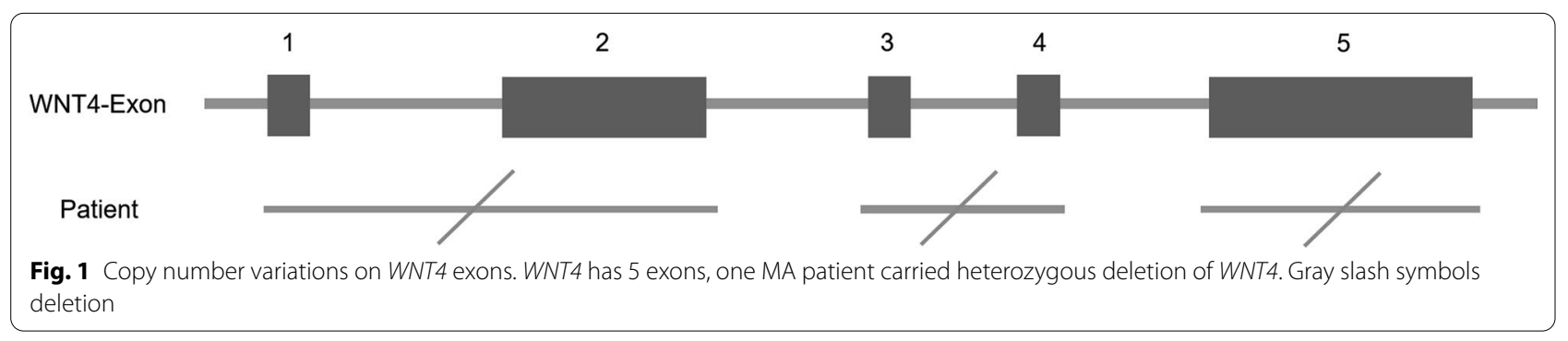




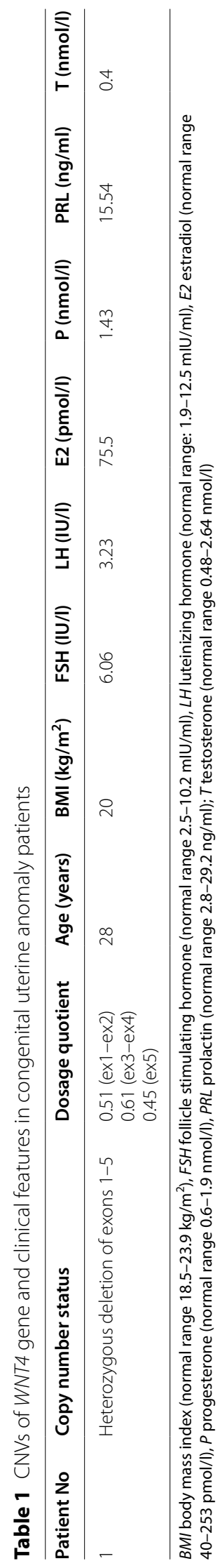




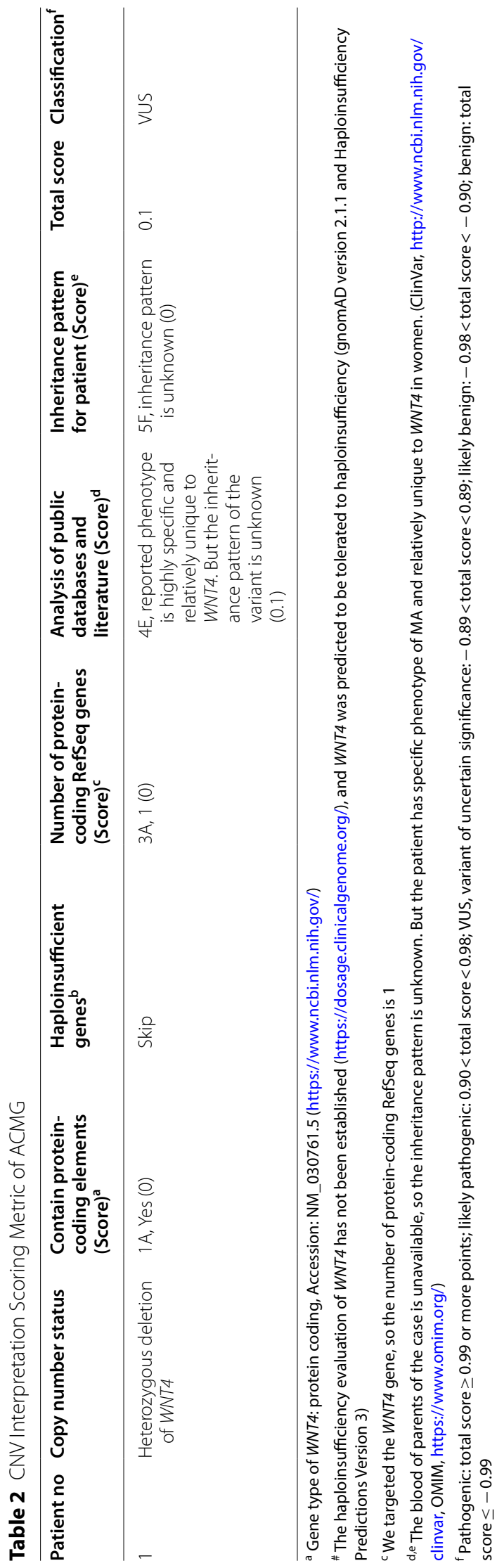




\section{Online database analysis}

No identical overlapping variant was found in the DGV database and GnomAD database. Therefore, this heterozygous whole deletion of WNT4 was considered to be rare in the general population. In the OMIM database, the mutation of WNT4 was reported to be associated with abnormal Müllerian ducts in women. The heterozygous deletion of WNT4 was considered variant of uncertain significance (VUS) according to the ACMG guidelines (see Table 2 for details).

\section{Discussion}

WNT4 has five exons and is located at 1p36.12 [genome coordinates (GRCh38): 1:2217307-22143980] (NCBI, https://www.ncbi.nlm.nih.gov/). It is a member of the WNT family of signaling molecules. The signaling protein encoded by this gene plays an important role in early embryonic sex determination, nephrogenesis, and female reproductive tract development $[6,13]$. WNT4 ${ }^{-1-}$ female mice have been reported to show the phenotypes of sex reversal, Müllerian duct aplasia, hyperandrogenism, and follicle depletion $[6,14]$. WNT4 $4^{\text {monomeric cherry/monomeric- }}$ cherry mice were shown to form Müllerian ducts in only $45 \%$ of cases [20].

The abnormal Müllerian ducts caused by WNT4 mutation are rare, but are pathological. Four missense mutations of WNT4 (p.L12P, p.E226G, p.R83C, p.A233) were found in four unrelated women with MA between 2004 and 2011 [15-18]. However, Chang et al. did not find any significant WNT4 mutation in 189 Chinese women with MA in 2012, although they did not analyze the CNVs [21]. Meanwhile, we identified only one heterozygous deletion in WNT4 in a single female patient among 248 Chinese women affected by MA. This shows the rarity of CNVs in MA patients. To our best knowledge, it is the first report of CNVin WNT4 in MA patients overall. And the deletion was assessed as VUS. Only missense variants have so far been associated with the phenotype, so that loss-of-function is not a known mechanism of disease. A VUS is not a benign or likely benign variant, the deletion we observed may possibly contributed to MA. So further evidence might accumulate future recognition of $\mathrm{LOH}$ as a pathogenic mechanism.

We also found that the abnormality of the Müllerian duct caused by missense mutation of WNT4 differed from that caused by CNVs of WNT4. All of the women carrying the missense mutation showed Müllerian duct abnormalities (two patients showed absent vagina and uterus, while one 15-year-old patient showed uterine agenesis), hyperandrogenemia, and primary amenorrhea [15-18]. However, our patient with a septate uterus showed normal menstruation and testosterone (T). WNT4 inhibits steroidogenic enzymes by upregulating $D A X 1$. Steroidogenic enzymes [including 3b-hydroxysteroid dehydrogenase 2 (HSD3B2) and 17a-hydroxylase (CYP17A1)] are necessary for testosterone production. The four missense mutations found in previous studies have been proven to increase the levels of HSD3B2 and CYP17A1 in cells transfected with the mutant alone or in combination with the wildtype protein. It was concluded that mutation of WNT4 showed a dominant negative effect [15-18]. The loss of heterozygosity $(\mathrm{LOH})$ in the patient that we identified may only led to haploinsufficiency. Therefore, we believe that the phenotype caused by LOH of WNT4 might be less severe than that caused by a dominant negative effect.

In addition, some rearrangements of other genes or regions found in MA patients should not be overlooked. Some of these regions have been shown to be clinically significant, such as 1q21.1, 7p14.3, 16p11.2, 17q12, and 22q11. In addition, some candidate genes have been proposed, including $L H X 1, B B S 9, H N F 1 B$, and $T B X 6$ [22-25].For example, Bernardini et al. identified deletion of 17q12 (including LHX1) in two cases with MayerRokitansky-Kuster-Hauser (MRKH) syndrome [22]. Ledig et al. observed 22 CNVs (including in LHX1and $H N F 1 B)$ at regions 1q21.1, 17q12, and 22q11.21 in 15 of 48 patients with MRKH [24]. Meanwhile, Gervasini et al. found SHOX duplications at chromosomal region $\mathrm{Xp} 22$ in 5 of $30 \mathrm{MRKH}$ patients [26]. Thus, some other genes or regions should be noted in CNV analysis of MA patients.

\section{Conclusions}

One heterozygous deletion in WNT4 was identified by MLPA in a single female patient among 248 Chinese women affected by MA. This study reports CNVs of WNT4 in a large sample of MA patients from the Chinese population for the first time. This suggests that CNVs of WNT4 cannot be excluded in the occurrence of MA. This provides a genetic basis for precise treatment in the future.

\section{Abbreviations}

CNVs: Copy number variations; WNT4: Wingless-type MMTV integration site family, member 4; MA: Müllerian anomalies; MLPA: Multiplex ligation-dependent probe amplification; ESHRE/ESGE: European Society of Human Reproduction and Embryology/European Society for Gynaecological Endoscopy; U:

Uterine body; C: Cervix; V: Vagina; DQ: Dosage quotient; ACMG: American College of Medical Genetics and Genomics; DGV: Database of Genomic Variants; GnomAD: Genome Aggregation Database; OMIM: Online Mendelian Inheritance in Man; E2: Estradiol; T: Testosterone; TCIS: Transcervical incision of septa; HSD3B2: 3B-hydroxysteroid dehydrogenase 2; CYP17A1: 17A-hydroxylase; LOH: Loss of heterozygosity; MRKH: Mayer-Rokitansky-Kuster-Hauser.

\section{Acknowledgements}

Not applicable. 


\section{Authors' contributions}

YZ: conceived and designed the experiments, and wrote the paper; RW: wrote the paper, performed the experiments, and analyzed and interpreted the data; YC: performed the experiments; $\mathrm{YH}$ : analyzed and interpreted the data; $\mathrm{TL}$ : performed the experiments; YC: conceived and designed the experiments, and contributed to reagents, materials, analysis tools, and data; BW: conceived and designed the experiments, contributed to reagents, materials, analysis tools, and data, and will handle correspondence at all stages of refereeing and publication, also post-publication. YZ and RW consider as joint First Authors. YC (caoyunxia6@126.com) and BW contributed equally to the research project and can be considered co-corresponding authors.

\section{Funding}

This project was supported by the Beijing Municipal Natural Science Foundation (Grant No7192150).

\section{Availability of data and materials}

All data were generated or analyzed during this study are included in this article.

\section{Declarations}

Ethics approval and consent to participate

Our research was approved by the Ethics Committee of the National Research Institute for Family Planning. All participants signed informed consent forms during blood sample collection and analysis.

\section{Consent for publication}

Not applicable.

\section{Competing interests}

All authors declare no conflict of interest.

\section{Author details}

${ }_{1}^{1}$ Reproductive Medicine Center, Department of Obstetrics and Gynecology, The First Affiliated Hospital of Anhui Medical University, 218 Jixi Road, Shushan, Hefei 230022, China. ${ }^{2}$ Anhui Province Key Laboratory of Reproductive Health and Genetics, Biopreservation and Artificial Organs, Hefei, China. ${ }^{3}$ Anhui Provincial Engineering Research Center, Anhui Medical University, Hefei, People's Republic of China. ${ }^{4}$ Graduate School of Peking Union Medical College, Beijing, China. ${ }^{5}$ Center for Genetics, National Research Institute for Family Planning, 12 Dahuisi Road, Haidian, Beijing 100081, China.

Received: 16 November 2020 Accepted: 21 May 2021

Published online: 07 June 2021

\section{References}

1. Acien P, Acien MI. The history of female genital tract malformation classifications and proposal of an updated system. Hum Reprod Update. 2011;17:693-705.

2. Ludwin A, Pfeifer SM. Reproductive surgery for mullerian anomalies: a review of progress in the last decade. Fertil Steril. 2019;112:408-16.

3. Chan YY, Jayaprakasan K, Zamora J, Thornton JG, Raine-Fenning N, Coomarasamy $A$. The prevalence of congenital uterine anomalies in unselected and high-risk populations: a systematic review. Hum Reprod Update. 2011:17:761-71.

4. Grimbizis GF, Gordts S, Di Spiezio SA, Brucker S, De Angelis C, Gergolet M, et al. The ESHRE-ESGE consensus on the classification of female genital tract congenital anomalies. Gynecol Surg. 2013;10:199-212.

5. Raga F, Bauset C, Remohi J, Bonilla-Musoles F, Simon C, Pellicer A. Reproductive impact of congenital Mullerian anomalies. Hum Reprod. 1997:12:2277-81.

6. Vainio S, Heikkila M, Kispert A, Chin N, McMahon AP. Female development in mammals is regulated by Wnt-4 signalling. Nature. 1999:397:405-9.

7. Mericskay M, Kitajewski J, Sassoon D. Wnt5a is required for proper epithelialmesenchymal interactions in the uterus. Development. 2004;131:2061-72.

8. Parr BA, McMahon AP. Sexually dimorphic development of the mammalian reproductive tract requires Wnt-7a. Nature. 1998:395:707-10.
9. Carroll TJ, Park JS, Hayashi S, Majumdar A, McMahon AP. Wnt9b plays a central role in the regulation of mesenchymal to epithelial transitions underlying organogenesis of the mammalian urogenital system. Dev Cell. 2005;9:283-92.

10. Heikkila M, Peltoketo $H$, Vainio S. Wnts and the female reproductive system. J Exp Zool. 2001;290:616-23.

11. Ottolenghi C, Pelosi E, Tran J, Colombino M, Douglass E, Nedorezov T, et al. Loss of Wnt4 and Foxl2 leads to female-to-male sex reversal extending to germ cells. Hum Mol Genet. 2007;16:2795-804.

12. Naillat F, Prunskaite-Hyyrylainen R, Pietila I, Sormunen R, Jokela T, Shan J, et al. Wnt4/5a signalling coordinates cell adhesion and entry into meiosis during presumptive ovarian follicle development. Hum Mol Genet. 2010;19:1539-50.

13. Stark K, Vainio S, Vassileva G, McMahon AP. Epithelial transformation of metanephric mesenchyme in the developing kidney regulated by Wnt-4. Nature. 1994:372:679-83.

14. Heikkila M, Prunskaite R, Naillat F, Itaranta P, Vuoristo J, Leppaluoto J, et al. The partial female to male sex reversal in Wnt-4-deficient females involves induced expression of testosterone biosynthetic genes and testosterone production, and depends on androgen action. Endocrinology. 2005;146:4016-23.

15. Biason-Lauber A, Konrad D, Navratil F, Schoenle EJ. A WNT4 mutation associated with Mullerian-duct regression and virilization in a 46, XX woman. N Engl J Med. 2004;351:792-8.

16. Biason-Lauber A, De Filippo G, Konrad D, Scarano G, Nazzaro A, Schoenle EJ.WNT4 deficiency - a clinical phenotype distinct from the classic Mayer-Rokitansky-Kuster-Hauser syndrome: a case report. Hum Reprod. 2007;22:224-9.

17. Philibert P, Biason-Lauber A, Rouzier R, Pienkowski C, Paris F, Konrad D, et al. Identification and functional analysis of a new WNT4 gene mutation among 28 adolescent girls with primary amenorrhea and mullerian duct abnormalities: a French collaborative study. J Clin Endocrinol Metab. 2008;93:895-900.

18. Philibert P, Biason-Lauber A, Gueorguieva I, Stuckens C, Pienkowski C, Lebon-Labich B, et al. Molecular analysis of WNT4 gene in four adolescent girls with mullerian duct abnormality and hyperandrogenism (atypical Mayer-Rokitansky-Kuster-Hauser syndrome). Fertil Steril. 2011;95:2683-6.

19. Riggs ER, Andersen EF, Cherry AM, Kantarci S, Kearney H, Patel A, et al. Technical standards for the interpretation and reporting of constitutional copy-number variants: a joint consensus recommendation of the American College of Medical Genetics and Genomics (ACMG) and the Clinical Genome Resource (ClinGen). Genet Med. 2020;22:245-57.

20. Prunskaite-Hyyrylainen R, Skovorodkin I, Xu Q, Miinalainen I, Shan J, Vainio SJ. Wnt4 coordinates directional cell migration and extension of the Mullerian duct essential for ontogenesis of the female reproductive tract. Hum Mol Genet. 2016;25:1059-73.

21. Chang X, Qin Y, Xu C, Li G, Zhao X, Chen ZJ. Mutations in WNT4 are not responsible for Mullerian duct abnormalities in Chinese women. Reprod Biomed Online. 2012;24:630-3.

22. Bernardini L, Gimelli S, Gervasini C, Carella M, Baban A, Frontino G, et al. Recurrent microdeletion at $17 q 12$ as a cause of Mayer-Rokitansky-KusterHauser (MRKH) syndrome: two case reports. Orphanet J Rare Dis. 2009;4:25.

23. Cheroki C, Krepischi-Santos AC, Szuhai K, Brenner V, Kim CA, Otto PA, et al. Genomic imbalances associated with mullerian aplasia. J Med Genet. 2008;45:228-32

24. Ledig S, Schippert C, Strick R, Beckmann MW, Oppelt PG, Wieacker P. Recurrent aberrations identified by array-CGH in patients with Mayer-RokitanskyKuster-Hauser syndrome. Fertil Steril. 2011;95:1589-94.

25. Sandbacka M, Laivuori H, Freitas E, Halttunen M, Jokimaa V, Morin-Papunen L, et al. TBX6, LHX1 and copy number variations in the complex genetics of Mullerian aplasia. Orphanet J Rare Dis. 2013;8:125.

26. Gervasini C, Grati FR, Lalatta F, Tabano S, Gentilin B, Colapietro P, et al. SHOX duplications found in some cases with type I Mayer-Rokitansky-KusterHauser syndrome. Genet Med. 2010;12:634-40.

\section{Publisher's Note}

Springer Nature remains neutral with regard to jurisdictional claims in published maps and institutional affiliations. 